\title{
A Case of Left Ventricular Myocardial Calcification in a Patient with Severe Sepsis
}

\author{
Carly Sedlock, MD, Randi Zukas, MD, and Hillary Landon, MD
}

\section{INTRODUCTION}

Sepsis is an important source of morbidity and mortality among patients admitted to the Intensive Care Unit (ICU) and can cause critical damage to all organ systems. Regarding cardiac complications, sepsis can acutely result in cardiomyopathy characterized by ventricular dilatation and a depressed ejection fraction. ${ }^{1}$ Myocardial calcification is a more rare, delayed cardiac complication of severe sepsis that has been reported in literature. ${ }^{1-6}$ Herein we report a case of a patient with Acute Myeloid Leukemia (AML) admitted to the $T J U H I C U$ requiring prolonged vasopressor support for severe sepsis whose clinical course was complicated by left ventricular myocardial calcification.

\section{CASE PRESENTATION}

A 56-year-old female with a history of AML status post $7+3$ induction chemotherapy, midostaurin and HIDAC consolidation presented to the emergency department with acute onset nausea, vomiting, and diarrhea. While in the ED, the patient was found to be in septic shock with fever, hypotension, tachycardia and tachypnea. Physical exam was notable for significant respiratory distress, altered mental status, diffuse wheezing and tachycardia with normal heart sounds. Labs were notable for an acute kidney injury with creatinine 2.6 (baseline 0.6), transaminitis, lactate of 10.5 , severe neutropenia, hemoglobin of 5.8, and platelets of 2,000. The patient was initiated on broad spectrum antibiotics and vasopressors, transfused with blood products, intubated for work of breathing and admitted to the medical Intensive Care Unit. The patient's hypotension was severe and persistent, and was thought to be due to both septic and cardiogenic shock. Septic shock was secondary to multifocal pneumonia, typhlitis complicated by E. coli bacteremia, VRE urinary tract infection, and CMV viremia; the patient was treated with meropenem, daptomycin and foscarnet. A TTE revealed new global hypokinesis with EF 35\% and the patient was started on dobutamine until her hypotension resolved. In the setting of her shock, the patient's hospital course was complicated by acute renal failure requiring temporary dialysis and vasopressor-induced ischemia with gangrene requiring bilateral finger transmetacarpal and bilateral toe transmetatarsal amputations. Weeks later, during the patient's prolonged hospital stay, she developed neutropenic fever and a CT chest was repeated which showed new diffuse myocardial attenuation involving the left ventricle (Figure 1). A repeat echocardiogram confirmed calcification of the papillary muscles as well as persistently decreased left ventricular systolic function with EF 35-40\%

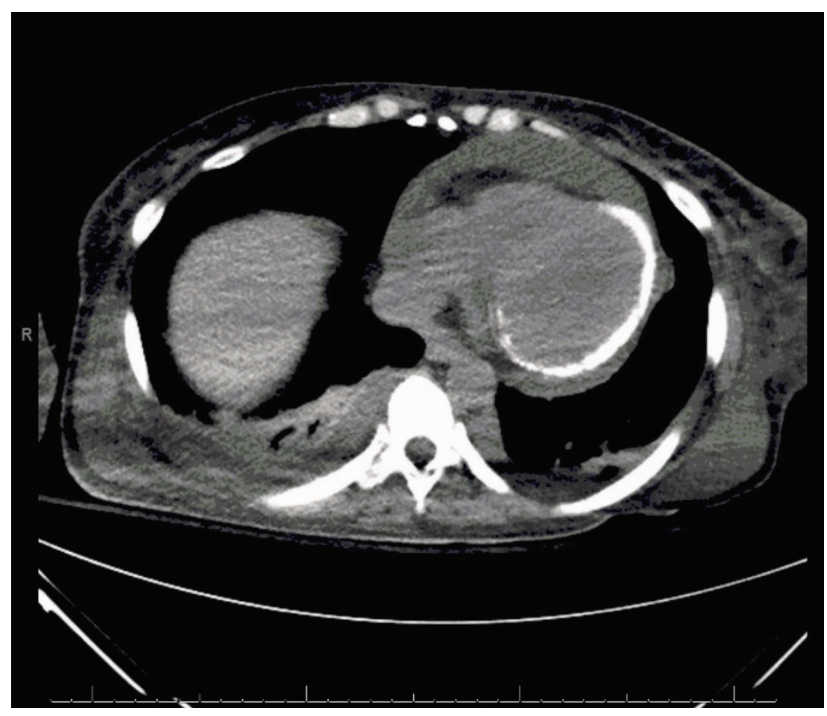

Figure 1: CT chest showing LV myocardial calcification.

\section{DISCUSSION}

In this paper, we present a patient with AML who developed left ventricular myocardial calcification several weeks after a prolonged ICU course requiring high dose vasopressor support for severe sepsis. While localized myocardial calcification may often be observed after myocardial infarction, diffuse myocardial calcification is a rare, serious complication of septic shock that is usually discovered, often incidentally on repeat imaging, several weeks after the patient's initial presentation. ${ }^{2.3}$ Calcium deposition in cardiac myocytes occurs via metastatic and dystrophic calcification. ${ }^{1,2}$ Metastatic calcification results from disturbances in calcium and phosphate metabolism, often in the setting of chronic renal failure. ${ }^{1,2}$ Dystrophic calcification occurs when calcium deposits into cardiac myocytes that have undergone necrosis, which occurs with myocardial infarction, myocarditis, trauma, cardiac surgery, and severe sepsis. ${ }^{2}$ Although the exact mechanism 
of sepsis-related dystrophic calcification is unclear, it is proposed that sepsis-induced circulatory changes, cytokines, and circulating myocardial depressant substances increase the permeability of cardiac myocyte membranes to calcium, resulting in calcium influx, calcium deposition, myofibrillar hypercontraction, and phosphate depletion; all these changes may cause mitochondrial dysfunction and myocardial necrosis., 1,4.5 In the setting of sepsis, high levels of catecholamines, vasopressors given for the treatment of hypotension, and acidosis also cause injury to cardiac myocyte membranes, promoting calcium deposition. ${ }^{6}$

The diagnosis of myocardial calcification is often discovered incidentally on thoracic CT obtained for another clinical indication, as occurred in this patient. CT is a highly sensitive and specific imaging modality for this diagnosis. ${ }^{1.5}$ Echocardiogram may show normal myocardium or increased echodensity of the ventricular walls, demonstrating calcification; systolic or diastolic dysfunction may also be observed. ${ }^{1}$ There are no clear therapies for prevention or treatment of this cardiac complication. ${ }^{2}$

This case highlights a rare but serious and delayed complication of severe sepsis that should be recognized by providers caring for critically ill patients. More data is needed to assess long term clinical consequences of this phenomenon and interventions for both prevention and treatment.

\section{REFERENCES}

1. Akbas T, Ozen M, Adiguzel C, Cimsit NC, Karakurt S. Cardiac Calcification Following Severe Sepsis: A Case Report and Review of the Literature. Anesth Clin Res. 2014:5(5):409

2. van Kruijsdijk R, van der Heijden J, Uijlings R, Otterspoor L. Sepsis-Related Myocardial Calcification. Images Case Reports Hear Fail. 2011:4:e16-e18.

3. Lapatto-Reiniluoto O, Vaalamo M, Takkunen, O MM. Left ventricular calcification following resuscitation. J Intern Med. 2000;248:85-87.

4. Furman MS, Healey TT, Agarwal S, Grand DJ, Atalay MK. " Heavy hearted havoc" - A case series of petrified myocardium. J Cardiovasc Comput Tomogr. 2018;12(6):e21-e23.

5. Schellhammer F, Ansen S, Arnold G, Brochhagen HG, Lackner K. Myocardial Calcification following Septic Shock. Cardiology. 2002;98:102-103.

6. Kapandji N, Redheuil A, Fouret P, Luyt C, Cluzel P, Combes A. Extensive Myocardial Calcification in Critically Ill Patients. 2018;46(7). 